\title{
Configuration Discussions of the Chemically Recuperated Gas Turbine Powering a Ship
}

\author{
Fumin Pan ${ }^{1}$, Hongtao Zheng ${ }^{1}$, Pingping Luo ${ }^{2}$, Ren Yang ${ }^{1}$ \\ ${ }^{1}$ College of Power and Energy Engineering, Harbin Engineering University, Nantong Street, \\ NO.145, Harbin, 150001, China \\ ${ }^{2}$ China Shipbuilding Industry Corporation, NO. 703 Institute, China
}

Keywords: Chemically recuperated gas turbine; Configuration determination; Diesel-steam reforming; Marine propulsion; Performance analysis.

\begin{abstract}
The chemically recuperated gas turbine (CRGT) is a promising engine on ship because of its high thermal efficiency and low pollutant emission. This paper is aimed at determining an applicable configuration of the CRGT for marine applications. Diesel is the selected fuel, and the minimization of Gibbs free energy method (MGFE) is applied to model the diesel-steam reforming reaction. A chemical regenerator (CR) is designed based on the reforming reaction, and a steam generator (SG) is set on the foundation of thermodynamics. Some feasible configurations of the CRGT are proposed based on a marine gas turbine with two spool shaft and a free power turbine and the best one is determined by performance calculation. The best configuration has a $44.51 \%$ thermal efficiency at rated 25MW output power of the prototype, and good stability in operation. Additionally, the selected configuration has lower combustion temperature at the same output power with the other configurations, and it can yield less NOX emissions. These works form the foundation of the CRGT being applied in the marine propulsion and provide a research thought for the CRGT in other applications.
\end{abstract}

\section{Introduction}

Due to the lower weight and space requirement for the prime mover system and lower amounts of pollutant emissions, the gas turbine is being widely used in marine propulsion; and it offers other advantages such as high specific power, rapid start-up, good manoeuvrability and low noise. However, the gas turbine has the disadvantages such as low thermal efficiency and poor part-load performance. For these reasons, advanced configurations are proposed such as the combined cycle [1], the chemically recuperated gas turbine (CRGT), and so on. The CRGT is based on fuel-steam reforming and recovery of heat from the waste gas. These steps improve the fuel quality and the energy utilization ratio, and reduce the pollutant emissions.

In 1992, Janes discussed the research on compressor inter-cooling and turbine reheating [2]. Then, various configurations based on the above ones are proposed, and thermodynamic analyses are performed to verify their advancement by Abdallah [3] and Carapellucci [4]. These studies confirm the advantages of the CRGT in power generation. Nowadays, researches for the CRGT practical applications have been carried out. In 2010, Zheng presented an efficient steam generator and evaluated the CRGT performance [5]. These studies verified the application prospect of the CRGT. Among others, almost all these schemes are built based on gas turbine in power plant, and the CRGT applying on ship has remained elusive. However, these researches provide the basic insights for solution determination of the CRGT.

Another active area of the CRGT research is the theoretical and experimental studies of steamreforming mechanism and designing a reforming regenerator base on the mechanism. During 20032006, Nagasaki studied steam reforming of methane and methanol, and a developed reforming regenerator using the methanol as the fuel [6-7]. In 2008, Huan-Liang Tsai designed a reforming regenerator by using ethanol-steam reforming in a thermal plasma reformer [8]. These reactions produce deeper reaction degree when heated by the waste gas from the turbine; however, ethanol is 
not as accessible as methane. These studies help in selecting catalysts, designing of reforming regenerators, and modelling of the reforming process.

This work is in continuation with the earlier research studies as mentioned above, and it aims at promoting the practical application of the CRGT for marine propulsion. The minimization of Gibbs free energy method is adopted in modelling diesel-steam-reforming. The chemical reformer and the steam generator are designed under thermodynamic principles. Upon the above works, some configurations of the CRGT based on a marine gas turbine are proposed, and performances of these configurations are calculated to determine which the best choice is. It is believes that all the works will form the foundation of the research of the CRGT on ship.

\section{Calculation Models}

\section{Overall Model}

The prototype in this study is a marine gas turbine (GT) with two spool shafts and a free power turbine. The performance parameters of the selected gas turbine at design point are listed in Table 1.

Table 1 Performance parameters of the GT at design point

\begin{tabular}{ccccc}
\hline $\mathrm{Ne}(\mathrm{MW})$ & $\eta(\%)$ & $\mathrm{Gf}(\mathrm{kg} / \mathrm{s})$ & TCB $(\mathrm{K})$ & Teg $(\mathrm{K})$ \\
\hline 25 & 35.85 & 1.625 & 1528.8 & 790.7 \\
\hline
\end{tabular}

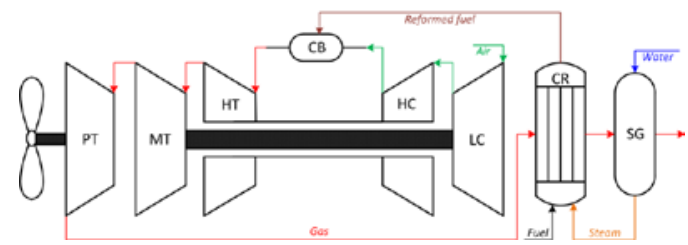

Fig.1.Schematic diagram of the S-CRGT

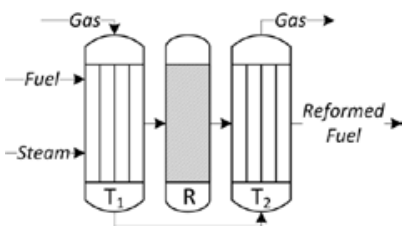

Fig.2.Calculation model of CR

A simple CRGT (S-CRGT) configuration is shown in Fig. 1. The waste gas from the PT flows first into the chemical reformer (CR) to provide heat for the diesel-steam reforming. Next, it flows into the steam generator (SG) to yield steam for reforming and fresh water supply on ship. And the reformed fuel is fed into the combustor (CB) as the energy source.

The schematic diagram of the CR is shown in Fig. 2, with the hypothesis that the fuel and steam reform adiabatically (R) after blending and being preheated by the waste gas first in the heat exchanger $T_{1}$, and then the reformed fuel is heated again by the waste gas in the heat exchanger $T_{2}$.

All the energy fed into the combustor which consists of the energy carried by the fuel and that recovered from the exhaust gas can be calculated as the following Equation 1. The total energy can be considered as the corrected heat value of the given fuel.

With the hypothesis that the products of the diesel-steam reforming consists of $\mathrm{C}, \mathrm{CO}, \mathrm{CO}_{2}, \mathrm{H}_{2}$, $\mathrm{CH}_{4}$ and $\mathrm{H}_{2} \mathrm{O}$, the energy carried by the reformed gas out of the CR can be described as the corrected heat value of the feeding fuel as Equation 1.

$$
h_{u, f}^{\prime}=\left(n_{\mathrm{CH}_{4}} M_{\mathrm{CH}_{4}} h_{u, \mathrm{CH}_{4}}+n_{\mathrm{CO}} M_{\mathrm{CO}} h_{u, \mathrm{CO}}+n_{\mathrm{H}_{2}} M_{\mathrm{H}_{2}} h_{u, \mathrm{H}_{4}}+m_{r f} h_{r f}\right) / n_{f} M_{f}
$$

\section{Modeling of the CR}

The modelling of the CR is to develop calculation models of the heat exchangers and the reformer, which are described below. The simulation of heat exchanger is based on heat transfer and thermal equilibrium equations.Values of $K$ and $F$ are given in the Section Design of the $C R$. In the simulation, $F$ is held constant and $K$ is directly proportional to the mass flow rate. The value of $\eta$ for all the heat exchangers is fixed at 0.99 .

The MGFE method is used to develop the mathematic model of the reformer by the criterion that the Gibbs free energy of the system reaches the minimization [9]. A thermodynamic model is developed without considering the catalysts and practical reaction conditions.

If the molecular formula of the fuel is assumed as $\mathrm{C}_{\mathrm{x}} \mathrm{H}_{\mathrm{y}} \mathrm{O}_{\mathrm{z}}$, the production of the products will be $\mathrm{C}, \mathrm{CO}, \mathrm{CO}_{2}, \mathrm{H}_{2}, \mathrm{CH}_{4}$ and $\mathrm{H}_{2} \mathrm{O}$. The chemical equation to descript this process is as Equation 2.

$$
\mathrm{C}_{\mathrm{x}} \mathrm{H}_{\mathrm{y}} \mathrm{O}_{\mathrm{z}}+a \mathrm{H}_{2} \mathrm{O} \rightarrow b \mathrm{C}+c \mathrm{CO}+d \mathrm{CO}_{2}+e \mathrm{H}_{2}+f \mathrm{CH}_{4}+g \mathrm{H}_{2} \mathrm{O}
$$


The Gibbs energy of the system can be described as Equation 3,

$$
G^{T}=\sum_{i=1}^{N}\left(n_{i} \mu_{i}\right)
$$

For real gas, $u_{i}$ can be calculated as Equation 6, in which molar $f$ is fugacity,

$$
u_{i}=G_{i}^{0}+R T \ln \left(\frac{f_{i}}{f_{i}^{0}}\right)
$$

Thus, Equation 5 transforms to Equation 5,

$$
G^{T}=\sum_{i=1}^{N}\left(n_{i} G_{i}^{0}\right)+\sum_{i=1}^{N}\left(n_{i} R T \ln \left(\frac{f_{i}}{f_{i}^{0}}\right)\right)
$$

The balancing process converts to a nonlinear optimization to acquire the minimization of Equation 5, and the constrained conditions are described as follows:

Element balance constrained conditions,

$$
\begin{aligned}
& \mathrm{C}: x=b+c+d+f \\
& \mathrm{H}: y+2 a=2 e+4 f+2 g \\
& \mathrm{O}: z+a=c+2 d+g
\end{aligned}
$$

And non-negative constrained conditions,

$$
b, c, d, e, f \geq 0 \text {. }
$$

\section{Design of the CR}

\section{Experiment Confirmation of the MGFE in Methane-Steam Reforming}

The calculation results by the MGFE method and the experiment data by non-thermal plasma catalyst in literature [10] are shown in Fig. 3. Here is the experiment condition: 673K, ISO atmospheric pressure, $\mathrm{m}$ (molar ratio of $\mathrm{H}_{2} \mathrm{O}$ to $\mathrm{C}, 2$ to 5).

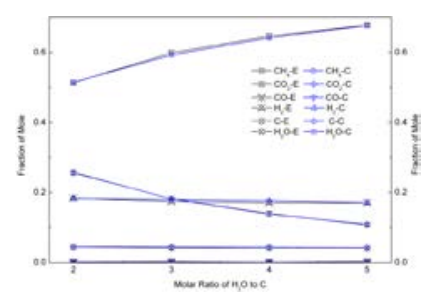

E: experiment C: calculation

Fig.3 Products of methane-steam reforming

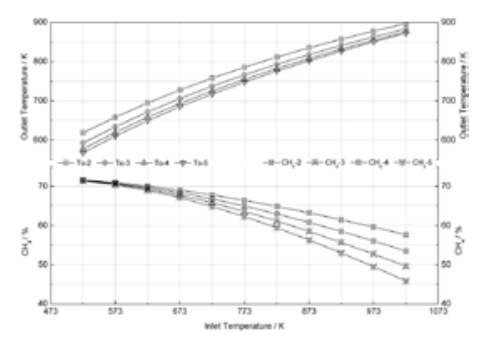

Fig.4 Methane selectivity of diesel-steam reforming

Table 2 Difference analysis of experiment \& calculation

\begin{tabular}{ccccc}
\hline $\mathrm{m}$ & $\mathrm{CH} 4$ & $\mathrm{CO} 2$ & $\mathrm{H} 2$ & $\mathrm{H} 2 \mathrm{O}$ \\
\hline 2 & $0.48 \%$ & $1.04 \%$ & $0.63 \%$ & $0.22 \%$ \\
3 & $0.17 \%$ & $4.75 \%$ & $3.44 \%$ & $1.07 \%$ \\
4 & $1.05 \%$ & $2.76 \%$ & $3.08 \%$ & $0.78 \%$ \\
5 & $1.58 \%$ & $2.62 \%$ & $0.37 \%$ & $0.17 \%$ \\
\hline
\end{tabular}

The main products differences of the experiments and calculations are listed in Table 2. $\mathrm{CO}_{2}$ has the biggest difference $4.75 \%$. However, the molar fraction of $\mathrm{CO}_{2}$ is very small. The differences of the other products are under $3.44 \%$. Either in the calculations or experiments, the molar fraction of $\mathrm{C}$ and $\mathrm{CO}$ is very small. 
It can be concluded that the MGRE method are well used in modelling the methane-steam reforming based on the results illustrated in Fig.3 and Tables 2.

\section{Calculation of the Diesel-Steam Reforming by the MGFE}

The selected molecular formula of diesel is $\mathrm{C}_{10} \mathrm{H}_{18}$. The MGFE method are used to calculate the diesel-steam reforming under the conditions that pressure is $2.5 \mathrm{MPa}$, the value of $m$ is from 2 to 5 , and the temperature at the entrance of the $\mathrm{R}$ is from 523.15 to $1023.15 \mathrm{~K}$. The results are shown in Fig. 2.

The selectivity of $\mathrm{CH}_{4}$ in the products calculated as Equation 10, and it describes the reaction depth.

$$
\mathrm{CH}_{4} \%=\frac{n_{\mathrm{CH}_{4}}}{n_{\mathrm{CH}_{4}}+n_{\mathrm{C}}+n_{\mathrm{CO}}+n_{\mathrm{CO}_{2}}} \times 100 \%
$$

The results show that the temperature and mole fraction of the products in the $\mathrm{R}$ are mainly influenced by the inlet temperature and the value of $m$. There are two conclusions: i) the temperature of the products decreases as the value of $m$ rises and the temperature of the entrance are fixed at the same value. This illustrates that the reaction absorbs more heat and the temperature of the products decreases when the value of $m$ increases. As a result, the reaction proceeds deeper and the $\mathrm{CH}_{4}$ fractions decrease. ii) The temperature of the products increases as the temperature of the entrance rises and the value of $m$ is fixed at the same value. This illustrates that higher temperature promotes the reaction and then the $\mathrm{CH}_{4}$ fractions decrease. It can be concluded that a higher value of the inlet temperature and $m$ can promote the steam reforming reaction.

\section{Thermodynamic Design of the CR}

The CR is designed according to the thermodynamics principle based on the exhaust gas parameters of the selected gas turbine.

(1)Thermodynamic Design of the $T_{1}$. It is illustrated that a satisfactory value of $m$ is 4 in Fig. 4 and the previous researches confirm this [3, 4]. A high value of the $\mathrm{m}$ can bring better reforming performance but excessive request of steam. And the fuel mass flow rate is set at $1.5 \mathrm{~kg} / \mathrm{s}$ with the consideration that the CRGT would produce more gas because of steam injection and more air induction. Assume the working conditions of the gas is that the temperature is $573.15 \mathrm{~K}$, and the pressure is $2.5 \mathrm{MPa}$ at the entrance based on the performance of the prototype at the design point. The results of thermodynamic design of $\mathrm{T}_{1}$ are that $\mathrm{K}$ is $400 \mathrm{~W} /\left(\mathrm{m}^{2} \cdot \mathrm{K}\right)$ [11], $\mathrm{F}$ is $87.26 \mathrm{~m}^{2}$.

(2) Thermodynamic Design of the $T_{2}$. The temperature of the products at the exit of the $R$ is 693.34 $\mathrm{K}$ which is acquired by the MGFE based on the parameters after the design of the Section Thermodynamic Design of the $T_{1}$. This temperature is almost equal to the temperature of the gas out of the $T_{1}$. So, there is no need to set the $T_{2}$. Thus, the CR consists of the $T_{1}$ and $R$.

Then the heat transfer coefficient of the SG is selected as $1000 \mathrm{~W} /\left(\mathrm{m}^{2} \cdot \mathrm{K}\right)$ [13], and the heat transfer area can be calculated. The value of the heat transfer area is $239.28 \mathrm{~m}^{2}$.

\section{Configuration Determination of the CRGT on ship}

Here, configurations of the CRGT are constructed and performance parameters at the same output power with that of the prototype at the design point.

\section{Simple CRGT}

The simple CRGT is shown in Fig.1. When the output power of the simple CRGT is 25 MW which is the same with that of the prototype at the design point, the temperature of the gas out of the gas turbine is $574 \mathrm{~K}$ which cannot maintain a mentionable reaction degree of the diesel-steam reforming. The performance parameters are calculated when the output power of the S-CRGT is $25 \mathrm{MW}$ and listed in Table 3. Then, advanced configurations of the CRGT should be proposed to maintain a higher gas temperature at the entrance of the CR to boost the diesel-steam reforming process. These configurations will be discussed in the following Sections Back-Reheating CRGT and MiddleRecovering, and performance parameters will be listed at the $25 \mathrm{MW}$ output power. 
Table 3 Main performance parameters of the S-CRGT at design point

\begin{tabular}{cccccc}
\hline Ne $(\mathrm{MW})$ & $\eta(\%)$ & Gf $(\mathrm{kg} / \mathrm{s})$ & TCB $(\mathrm{K})$ & $\mathrm{Tr}, \mathrm{i}(\mathrm{K})$ & $\mathrm{Teg}(\mathrm{K})$ \\
\hline 25 & 42.12 & 1.38 & 1209 & 574 & 376 \\
\hline
\end{tabular}

\section{Back-Reheating CRGT}

Reheating the gas by combusting a fraction of fuel is a method to elevate the gas temperature at the entrance of the CR. A configuration is constructed here as shown in Fig. 5. The configuration is called back-reheating CRGT (BR-CRGT) in which a re-combustor is set between the (power turbine) PT and CR. A fuel allocation coefficient called $d$ which is the ratio of the fuel fed into to the CR to all the fuel fed into the system is introduced. To acquire the best thermal efficiency of each system, the value of $d$ for this system is set at 0.8 according performance calculations. The performance parameters are calculated when the output power of the BR-CRGT is $25 \mathrm{MW}$ and listed in Table 4.

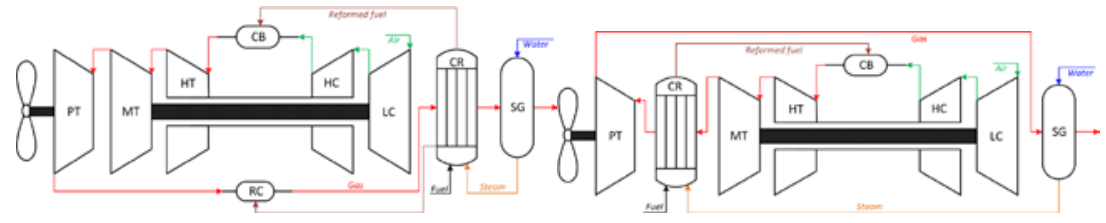

Fig. 5 Schematic diagram of the BR-CRGT

Fig. 6 Schematic diagram of the MR-CRGT

Table 4 the performance parameters of the BR-CRGT at design point

\begin{tabular}{cccccc}
\hline Ne (MW) & $\eta(\%)$ & Gf (kg/s) & TCB (K) & Tr,i (K) & Teg (K) \\
\hline 25 & 43.73 & 1.67 & 1215.21 & 688.14 & 433.78 \\
\hline
\end{tabular}

Middle-Recovering CRGT (MR-CRGT)

There is another scheme to boost the inlet gas temperature of the CR that the CR is allocated between the middle pressure turbine (MT) and PT as shown in Fig. 6, called the middle-recovering CRGT (MR-CRGT). This configuration has its feasibility because of the flexibility of the PT allocation of and the high gas temperature at the exit of the MT. The performance parameters are calculated when the output power of the MR-CRGT is 25MW and listed in Table 5.

Table 5 the performance parameters of the MR-CRGT at design point

\begin{tabular}{cccccc}
\hline $\mathrm{Ne}(\mathrm{MW})$ & $\eta(\%)$ & Gf $(\mathrm{kg} / \mathrm{s})$ & $\mathrm{TCB}(\mathrm{K})$ & $\mathrm{Tr}, \mathrm{i}(\mathrm{K})$ & $\mathrm{Teg}(\mathrm{K})$ \\
\hline 25 & 44.51 & 1.32 & 1219.33 & 753.52 & 389.23 \\
\hline
\end{tabular}

\section{Performance Calculations of the CRGTs}

\section{Performance Discussion of the CRGTs}

With the assumption that all these configurations are used in the same condition (ISO standard atmosphere), the performance parameters change with the output power and the relationship curves are plotted in the following Figures 8-11.

(1) Economy of these configurations. The thermal efficiency and the fuel consumption are the parameters to assess the economy of a system. Variations of the thermal efficiency and fuel supply for all the configurations in this paper are plotted in Fig. 7 and 8 respectively. It is shown that the MR-CRGT has the best economy in all the working conditions, the S-CRGT second and the BRCRGT lowest. The MR-CRGT and S-CRGT recover the heat of the gas from the PT and elevate the thermal efficiency. The BR-CRGT has low thermal efficiency because a part of the feeding fuel provides the energy to elevate the temperature of the gas from the PT, though it recovers the heat of the exhaust gas. The MR-CRGT set the chemical recover between the MT and PT. Two factors influencing the economy of the system appear here, i) the CR absorbs the heat from the gas which may cause the system yield less output power and demean the thermal efficiency; ii) the high inlet gas temperature of the CR can enhance the diesel-steam reforming, and it can elevate the thermal 
efficiency. The overall effect of the two influence factors is that the thermal efficiency of the MRCRGT is the highest of all the promoted configurations.

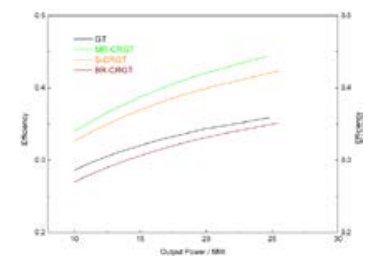

Fig. 7 Variations of $\eta$ with output power

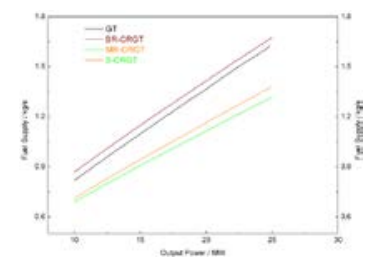

Fig. 8 Variations of $\eta$ with output power

(2) The outlet temperature of the CB. For the same configuration, the higher the combustion temperature is, the higher thermal efficiency the gas turbine system has. However, the high temperature causes two main problems: i) the complicated constructions of the combustor are needed to prevent to burn the shell of the flame tube; ii) the higher the combustion temperature is, the more $\mathrm{NO}_{\mathrm{X}}$ can be produced. The combustion temperatures of all the configurations are plotted in Fig. 9. The MR-CRGT and S-CRGT bring down the combustion temperatures, and has higher thermal efficiency. The BR-SCRGT also has a lower combustion temperature while the thermal efficiency is lower than that of the GT. It can be concluded that the CRGTs are environmentally friendly.

In the gas turbine system, the high-temperature exhaust gas brings out much heat which makes the system has poor economy. The MR-CRGT and S-CRGT have low exhaust gas temperatures because i) they have lower combustion temperatures, and ii) the CRs recover heat of the exhaust gas. It is shown that the variations of exhaust gas temperature with output power in Fig. 10.

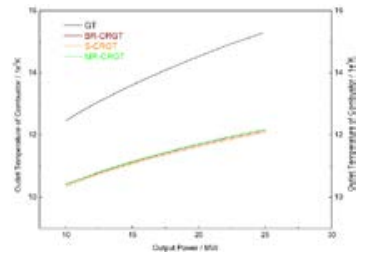

Fig. 9 Variations of $\mathrm{T}_{\mathrm{CB}}$ with $\mathrm{Ne}$

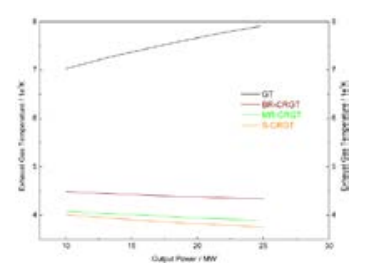

Fig.10 Variations of $\mathrm{T}_{\text {eg }}$ with $\mathrm{Ne}$

\section{Conclusions}

Determining the configuration of the CRGT on a ship forms the foundation of its application in marine propulsion system. The diesel-steam reforming is the adopted chemical reaction, and the minimization of Gibbs free energy method is used to model this process. A marine gas turbine with two spool shafts and a free power turbine is used as the prototype in this study and the CR is designed. Three configurations of the CRGT are proposed, and they are S-CRGT, BR-CRGT and MR-CRGT. Performances of these configurations under variable working conditions are calculated. It is suggested that the MR-CRGT should be the selected configuration for further researches because of the best economy, much less $\mathrm{NO}_{\mathrm{X}}$ emissions. Other conclusions can be acquired.

Diesel steam reforming. The minimization of Gibbs free energy method is acceptable in the modelling as the method is verified by methane-steam reforming, in which it shows high calculation precision. And experimental researches of the diesel-steam reforming should be the next research direction to refine the modelling of this reaction.

Performance under the low working conditions. The CRGTs show satisfactory performances under the low working conditions. The MR-CRGT and the S-CRGT have high thermal efficiency 
even under low working conditions. For example, the MR-CRGT has 34\% thermal efficiency at 10 MW output powers, which is $40 \%$ of that at the design working condition. The CRGTs also have good stability under low working conditions than the prototype.

\section{Acknowledgements}

Fumin Pan, Hongtao Zheng and all the other authors acknowledge the contribution of the staff's advices, and also appreciate the authors of the following references.

\section{References}

[1] F. Hoagland, A review on the use of gas and steam turbine combined cycles as prime movers for large ships, part I: background and design, Energy Convers Manage, 49 (12), p. 3458-67, 2008.

[2] J. Janes, Chemically recuperated gas turbine, California Energy Commission Staff Report, 1992.

[3] H. Abdallah, S. Harvey, Thermodynamic analysis of chemically recuperated gas turbines, Int. J. Therm. Sci, 40, p. 372-384, 2001.

[4] R. Carapellucci, A. Milosz, Thermodynamic optimization of a reheat chemically recuperated gas turbine, Energy Conversion and Management, 46, p.2936-2953, 2005

[5] H.T. Zheng, Y.L. Zhang, and R. Yan, Simulation research of the CRGT cycle gas turbine performance, Journal of Aerospace Power, 27, p. 118-123, 2012.

[6] T. Nakagaki, T. Ogawa, H. Hirata, K. Kawamoto., Development of chemically recuperated micro gas turbine, Transactions of the Japan Society of Mechanical Engineers, 125 (1), p. 391-397, 2003.

[7] N. Takao, Y. Masahiko, W. Tsunenori, K. Koji, Suitability Evaluation of Steam-Reforming Catalyst for DME-Fueled, Transactions of the Japan Society of Mechanical Engineers, Part B, 18 (6), p. 1633-1640, 2006.

[8] H.L. Tsai, C.S. Wang, C.H. Lee, Hydrogen production in a thermal plasma hydrogen reformer using ethanol steam reforming, Journal of the Chinese Institute of Engineers, 31 (3), p. 417-425, 2008.

[9] P. Mathieu, R. Dubuisson, Performance analysis of a biomass gassier, Energy Conversion and Management, 43, p. 1291-1299, 2002.

[10] Q. Liu, H.T. Zheng, L. Cai, CFD Modelling of Exhaust Heat Recovery Using Methane Steam Reforming in Steam Reformer of Chemically Recuperated Gas Turbine, Journal of Convergence Information Technology, 23(7), p. 152-161, 2012.

[11] K.S. Ramesh, P.S. Dusan, Fundamentals of Heat Exchanger Design, 1st ed. Wiley, 2002. 